La Revue

des Droits

de l'Homme

\section{La Revue des droits de l'homme}

Revue du Centre de recherches et d'études sur les droits fondamentaux

Actualités Droits-Libertés | 2019

\title{
Une condamnation de la Russie par la Cour européenne des droits de l'homme pour détournement de pouvoir à l'égard d'un opposant politique
}

Maria Lesire, Esther Sebillotte et Lise Souque

\section{(2) OpenEdition}

Journals

Édition électronique

URL : http://journals.openedition.org/revdh/6206

DOI : $10.4000 /$ revdh.6206

ISSN : 2264-119X

\section{Éditeur}

Centre de recherches et d'études sur les droits fondamentaux

\section{Référence électronique}

Maria Lesire, Esther Sebillotte et Lise Souque, « Une condamnation de la Russie par la Cour européenne des droits de l'homme pour détournement de pouvoir à l'égard d'un opposant politique », La Revue des droits de l'homme [En ligne], Actualités Droits-Libertés, mis en ligne le 03 mars 2019, consulté le 19 avril 2019. URL : http://journals.openedition.org/revdh/6206 ; DOI : 10.4000/ revdh.6206

Ce document a été généré automatiquement le 19 avril 2019

Tous droits réservés 


\title{
Une condamnation de la Russie par la Cour européenne des droits de l'homme pour détournement de pouvoir à l'égard d'un opposant politique
}

\author{
Maria Lesire, Esther Sebillotte et Lise Souque
}

Le 5 mai 1998, la Fédération de Russie ratifiait la Convention de sauvegarde des Droits de l'Homme et des libertés fondamentales du Conseil de l'Europe ainsi que ses protocoles $\mathrm{n}$ - 1, 4, 7, 9, 10 et 11. Dix-neuf ans plus tard, la Cour européenne des Droits de l'Homme (CEDH) a rendu 305 arrêts à l'encontre de cet État fédéral, soit plus d'un quart des arrêts produits au total par cette Cour.

1 Le 7 février 2018, la Cour s'est prononcée sur une nouvelle affaire, Lashmankin et autres $c$. Russie $^{1}$, concernant 23 requérants de différentes régions, relative à la liberté de manifestation. Il y a quelques mois, le rapport 2017/2018 de l'ONG Amnesty International ${ }^{2}$ , dans sa partie relative à la Russie, rapportait que "les droits à la liberté d'expression, d'association et de réunion pacifique ont fait l'objet de restrictions supplémentaires et le droit d'être jugé dans des conditions équitables a fréquemment été bafoué.". Durant les années 2017 et 2018, des manifestations, les plus importantes que le pays ait connues ces dernières années, ont eu lieu un peu partout sur le territoire. Des centaines de manifestants pacifiques, de passants et de journalistes ont été arrêtés. Nombre d'entre eux ont été soumis à des traitements cruels, inhumains ou dégradants, placés de façon arbitraire en détention prolongée ou jugés dans des conditions inéquitables et condamnés à de lourdes amendes et à plusieurs jours de "détention administrative ».

2 En l'espèce, la Cour énonce que les restrictions de lieu et de temps imposées aux organisateurs des manifestations, ainsi que les restrictions imposées à ceux-ci s'agissant des modalités de déroulement de celles-ci, s'analysaient en une atteinte au droit des 
requérants à la liberté de réunion, ajoutant que ceux-ci n'avaient eu accès à aucun recours effectif leur permettant de contester ces restrictions.

3 Rappelons qu'au début de l'année 2018, la Cour constitutionnelle russe avait énoncé que le simple fait de participer à un rassemblement pacifique «non autorisé » ne constituait pas une infraction au titre de l'article 212.1 du Code pénal, qui sanctionne les violations répétées de la réglementation en matière de manifestations. La peine d'emprisonnement prononcée au titre de cet article contre Ildar Dadine, le prévenu, avait été réexaminée le 22 février ; la Cour suprême avait ordonné la libération de ce militant en se fondant sur la circonstance que celui-ci avait manifesté sans violence

En dépit de la décision de relaxe du militant, les tribunaux russes n'avaient pas suivi la jurisprudence constitutionnelle pour Alexei Navalnyy, condamné pour avoir participé à des rassemblements non autorisés. Ce dernier a par la suite contesté la décision jusqu'à épuiser les voies de recours internes et saisir la Cour de Strasbourg. À l'issue d'une longue procédure, la Grande Chambre a condamné la Fédération de Russie (requête n 29580/12 et quatre autres) le 15 novembre $2018^{3}$.

5 Dans cette affaire, le requérant n'est pas un citoyen lambda. En effet, Aleksey Anatolyevich Navalnyy, est une personnalité politique russe de premier plan. Avocat et militant anticorruption, il représente la figure principale d'opposition de Vladimir Poutine, en dépit des intimidations du Kremlin. L'ONG Amnesty International a d'ailleurs qualifié celui-ci, dans un communiqué, de "prisonnier de conscience qui n'a commis aucun crime". Monsieur Navalnyy avait été déclaré inéligible par la Commission électorale centrale russe vendredi 23 juin 2017. Cette dernière avait estimé qu'il n'avait pas le droit de se présenter en raison d'une condamnation en 2013. Alors qu'il était candidat aux élections municipales, l'intéressé avait été reconnu coupable d'un détournement de fonds de 400000 euros, au détriment d'une société publique d'exploitation forestière dans la région de Kiev. Depuis lors, Navalnyy dénonce un procès politique et une affaire montée par le Kremlin.

6 Le requérant fut arrêté à sept reprises en 2012 et 2014 à l'occasion de différents rassemblements publics et systématiquement condamné sur le fondement de l'article 20.2 du code des infractions administratives lors de sept arrestations pour manquement à la procédure établie de conduite des événements publics. Le principal opposant politique du président russe estime que son arrestation, sa détention et sa condamnation administrative à sept reprises en 2012 et 2014 ont violé ses droits et étaient motivées par des mobiles politiques.

7 La Cour s'est d'abord prononcée sur l'affaire Navalnyy le 2 février $2017^{4}$. Elle conclut à la violation des articles 5 et 11 mais ne recherche pas s'il y a eu violation de ces dispositions en combinaison avec l'article 18. Une évolution semble possible puisque le juge Keller, dans une opinion dissidente, a rappelé que bien que l'article 18 soit une disposition accessoire, elle protège un intérêt distinct qui aurait pu être envisagé. L'article 18 concerne la limitation de l'usage des restrictions aux droits. Il dispose que "Les restrictions qui, aux termes de la présente Convention, sont apportées auxdits droits et libertés ne peuvent être appliquées que dans le but pour lequel elles ont été prévues". C'est à travers l'arrêt de Grande Chambre du 15 novembre 2018 que les juges vont statuer sur la violation de cet article.

8 Du fait du faible nombre de précédents, les juges vont exercer une diligence accrue lorsqu'ils vont statuer sur les allégations de motifs illégitimes (Partie I). Cependant, il en 
résulte une condamnation plus vindicative que la jurisprudence antérieure à l'égard de la Russie (Partie II).

\section{1./ La mobilisation de l'article 18 : une diligence accrue des juges}

9 Suite à cette requête, les juges de la Grande Chambre ont jugé nécessaire d'examiner l'affaire sous l'angle d'une éventuelle violation de l'article 18 de la Convention européenne des droits de l'Homme, rarement invoqué. Ce faisant, la Cour est conduite à préciser et faire progresser sa jurisprudence relative à l'interprétation et au champ d'application de l'article 18 de la Convention européenne des Droits de l'Homme.

10 La première mention de cette disposition s'est trouvée dans un document préparé par la Conférence de hauts fonctionnaires sur les droits de l'homme tenue du 8 au 17 juin 1950 à Strasbourg. Il semblerait que l'article 18 tire son origine du droit administratif français. En effet, le Conseil d'État français avait pour habitude de rechercher les intentions des auteurs d'actes administratifs afin de voir si un dessein caché se dissimulait derrière le voile de la légalité apparente.

11 Sont cités dans l'arrêt Navalnyy les travaux préparatoires de la Convention relatifs à l'article $18^{5}$. Selon ces derniers “(...) Il est légitime et nécessaire de limiter, quelquefois même de restreindre, les libertés individuelles pour permettre à tous d'exercer paisiblement leur liberté à eux, et pour assurer la primauté de la morale, du bien général, du bien commun et de l'utilité publique. (...) Mais lorsqu'il intervient pour supprimer, restreindre, limiter les libertés au nom, cette fois, de la raison d'État, pour se protéger selon la tendance politique qu'il représente contre une opposition qu'il estime dangereuse, pour détruire les libertés fondamentales qu'il devrait être chargé de coordonner et de garantir, c'est contre l'intérêt général qu'il intervient. Alors, la législation qu'il édicte est contraire aux principes de la garantie internationale “.

Dans ce cadre, la restriction imposée à des droits ou libertés peut être nécessaire mais doit toutefois être justifiée par un motif d'intérêt général. Il est clair que l'objet de l'article 18 est d'interdire le détournement de pouvoir'.

En l'espèce, la Cour conclut à la violation de l'article 18 en combinaison avec les articles 5 et 11. En proclamant le "droit à la liberté », l'article 5 de la Convention vise la liberté physique de la personne. Il a pour but d'assurer que nul n'en soit dépouillé de manière arbitraire. L'article 11, quant à lui, protège la liberté de réunion et d'association. L'article 18 n'est pas une notion autonome, ce qui explique que sa violation soit invoquée en combinaison avec les articles 5 et 11, mais complète les clauses prévoyant des restrictions aux droits et libertés énoncés dans la Convention. Il convient de préciser que l'article 18 n'a pas souvent été invoqué et que, lorsqu'il l'a été, la Cour a très rarement conclu à une violation. Compte tenu du faible nombre de précédents, la Cour va exercer un contrôle approfondi lorsqu'elle va analyser les allégations de motifs illégitimes du requérant. En l'espèce, elle va opérer à un rappel de la jurisprudence relative à l'article 18 à travers la décision Merabishvili c. Géorgie de 20177. Cet arrêt porte sur l'arrestation et la détention provisoire d'un ancien ministre de Géorgie. Dans ce dernier, les juges ont précisé l'interprétation de l'article 18. En effet, la Cour va clarifier la façon d'interpréter et d'appliquer l'article 18 de la Convention lorsqu'une restriction poursuit plusieurs buts. L'article 18 complète les clauses prévoyant des restrictions aux droits et libertés énoncés 
par la Convention. La Cour considère qu'une restriction peut être compatible avec la disposition normative de la Convention qui l'autorise dès lors qu'elle poursuit un des buts énoncés par cette disposition et, en même temps, être contraire à l'article 18 au motif qu'elle vise principalement un autre but qui n'est pas prévu par la Convention, autrement dit au motif que cet autre but est prédominant. À l'inverse, si le but prévu par la Convention est le but principal, la restriction ne méconnaît pas l'article 18 même si elle poursuit également un autre but (\$ 305).

Dans l'arrêt commenté dans la présente lettre, la Cour examine le caractère nécessaire ou justifié de la restriction au droit du requérant, cherche à s'assurer que celle-ci a été prise sur le fondement de motifs pertinents et suffisants et, enfin si celle-ci a bien été proportionnée aux buts ou motifs pour lesquels elle est autorisée par la Convention. ${ }^{8} \mathrm{La}$ seule présence d'un but qui ne relève pas des buts énoncés de façon limitative dans un article prévoyant une restriction aux droits et libertés de la Convention, ne peut emporter violation de l'article 18. Il s'agit donc pour la Cour de s'attacher à rechercher si la décision des autorités n'est pas cantonnée à la poursuite d'un but légitime, mais également d'un but non admis par la Convention. Pour ce fait, les juges vont étudier les éléments en sa possession et apprécier en toute liberté la recevabilité, la pertinence et la valeur de chaque élément du dossier.

Dans cette décision, les juges considèrent qu'à la lumière des éléments ci-dessus, en particulier de la chronologie et de la physionomie des événements ${ }^{9}$ et au-delà de tout doute raisonnable, les restrictions imposées au requérant lors des cinquième et sixième épisodes poursuivaient un but inavoué, contraire à l'article 18 de la Convention.

\section{2./Une condamnation plus vindicative de la Russie}

Dans l'arrêt rendu par la Chambre le 2 février 2017, la Cour avait reconnu que "l'arrestation et la détention administrative du requérant avaient eu pour effet d'empêcher et de dissuader lui-même et d'autres personnes de participer à des rassemblements de protestation et de s'investir activement dans l'opposition politique" (\$52). Toutefois, constatant à cet égard une violation des articles 5 et 11, elle n'avait pas estimé nécessaire de rechercher s'il y avait eu violation de l'article 18 en combinaison avec ces deux articles.

16 Pourtant, comme en témoigne l'opinion dissidente ${ }^{10}$ formulée à l'occasion de cet arrêt, "l'article 18 a beau être une disposition accessoire" (\$2) ou du moins non autonome, elle protège un intérêt juridique distinct des articles 5 et $11:$ la prévention d'une "injustice spécifique, à savoir l'atteinte à des droits conventionnels pour laquelle des justifications légitimes sont improprement avancées comme prétexte afin de dissimuler des arrièrepensées".

Dans son arrêt de Grande chambre, la Cour énonce que le grief tiré de l'article 18 constitue un aspect fondamental de l'affaire. Ce faisant, celle-ci va distinguer à nouveau parmi les 7 épisodes mentionnés les épisodes 5 et 6 des autres épisodes. Elle revient notamment sur le sixième épisode ayant eu lieu le 24 février 2014, au cours duquel le requérant a été arrêté alors qu'il patientait parmi les personnes qui attendaient devant le tribunal pour assister à une audience publique. Selon elle, "la police a délibérément fendu la foule afin de mettre la main sur lui et de lui faire quitter les lieux alors que rien dans son comportement ou dans son apparence ne le distinguait des autres individus pacifiques qui patientaient tranquillement derrière le cordon policier" (\$170). Ce sont ces 
faits qui amènent la Cour à rejoindre la thèse du requérant selon lequel celui-ci aurait été personnellement ciblé en raison de son rôle majeur dans l'opposition politique et du caractère particulièrement médiatique de son arrestation. Le cinquième épisode était lui aussi emblématique de cette intention, dans la mesure où le requérant avait été arrêté pour avoir conduit une "marche", alors même qu'il ne faisait que quitter les lieux d'une manifestation statique, accompagnée de personnes sans avoir de contrôle sur celles-ci.

Ce sont les restrictions imposées par les autorités russes au cours de ces deux épisodes qui, selon la Cour, "poursuivaient un but inavoué, contraire à l'article 18 de la Convention, à savoir celui d'étouffer le pluralisme politique, qui est un attribut du "régime politique véritablement démocratique » encadré par la "prééminence du droit ", notions auxquelles renvoie le Préambule de la Convention"(\$175).

Concernant la violation de l'article 11, la Cour se montre également plus vindicative à l'égard des pratiques russes que dans l'arrêt de chambre de 2017 qui avait toutefois reconnu le caractère dissuasif des arrestations et détentions litigieuses sur l'opposition politique. Elle ajoute ainsi qu"'incontestablement, ces mesures risquaient gravement aussi de dissuader d'autres partisans de l'opposition ainsi que la population en général de participer à des manifestations et, plus généralement, à des débats politiques ouverts" (\$152). Elle souligne également le caractère médiatique de l'affaire qui renforcerait l'effet dissuasif de ces mesures pour la population.

20 La Cour se permet par ailleurs d'évoquer les lacunes de la législation russe sur le sujet. Après avoir affirmé que "le respect des règles régissant les réunions publiques ne doit pas devenir une fin en soi” (\$144), la Cour constate un “manquement persistant des autorités nationales à faire preuve de tolérance vis-à-vis des réunions non autorisées mais pacifiques et, plus généralement, à appliquer des critères conformes aux principes découlant de l'article 11 de la Convention" (\$148).

21 Au début de son arrêt, celle-ci rappelle en outre que le Comité des ministres continue à veiller à l'exécution de l'arrêt Lashmankin ${ }^{11}$. Dans ce cadre, à l'occasion de sa 1318ème réunion ayant eu lieu en juin 2018, celui-ci avait souligné les "développements positifs" de la législation relative à la procédure pour l'organisation et la conduite d'événements publics. Toutefois, il avait également affirmé le "besoin d'adopter rapidement des mesures complémentaires", se référant aux lignes directrices établies par la Commission de Venise ainsi que l'Organisation pour la Sécurité et la Coopération en Europe (OSCE), une organisation internationale ayant pour but de favoriser le dialogue et la négociation entre l'Est et l'Ouest.

Elle revient ensuite sur ces lacunes qu'elles présentent comme étant structurelles : suite à l'arrêt Lashmankin notamment, "les pratiques internes [...] ont perduré et il y a même eu des réformes législatives introduisant davantage de restrictions". Elle pointe aussi du doigt l'interprétation extensive de la notion de "réunion soumise à notification" qui, en droit russe, justifie le pouvoir d'ingérence des autorités nationales dans les "réunions publiques pacifiques ne générant ni «troubles » ni nuisances" (\$ 150). La Cour estime que les conditions de forme requises par la législation russe pour l'organisation de réunions pacifiques sont excessivement restrictives. La Cour pousse même son raisonnement jusqu'à se demander si, du fait des caractéristiques de la loi russe, l'exercice des voies de recours internes ne serait pas ineffectif.

23 En définitive, si l'apport de l'arrêt est de mettre ainsi en lumière l'existence d'une lutte arbitraire des autorités contre l'opposition politique en Russie, l'opinion dissidente de 
certains juges laisse à penser qu'il aurait été loisible à la Cour de se placer sur le terrain de l'article 17 en ce qui concerne l'argument tiré de l'abus de droit. L'opinion dissidente jointe $^{12}$ à l'arrêt évoque une approche qui aurait semblé plus appropriée à certains juges. Ceux-ci énoncent qu'il aurait été, à leurs yeux, plus juste de se placer sur le terrain de l'article 17 qui concerne l'abus de droit. Bien que n'ayant jamais été appliquée à la conduite d'un État, cette disposition pourrait selon ces juges "s'appliquer à tout acte ou toute activité de l'État « visant à la destruction des droits ou libertés reconnus » dans la Convention ou ses Protocoles" (\$7), protégeant ainsi les individus de dérives totalitaires.

Ainsi, les rédacteurs de l'opinion dissidente que si les juges s'étaient livrés à une analyse des faits au regard de l'article 17, la Cour aurait été libre de procéder à une analyse globale des événements, en les interprétant conjointement comme des manifestations d'un système plus global de limitation abusive des droits fondamentaux des justiciables par des moyens législatifs, administratifs ou judiciaires sans avoir à examiner chacun des cas au regard de la limitation de la liberté de réunion du requérant.

L'on peut toutefois émettre de sérieux doutes quant à l'exécution de l'arrêt par la Russie au vu du rapport de crise durable s'étant instauré entre cet État Membre et le Conseil de l'Europe ${ }^{13}$. Tout d'abord, en réponse à l'annexion de la Crimée par Moscou, le Conseil avait privé la Russie de ses droits de vote à l'assemblée parlementaire du Conseil de l'Europe (APCE). En guise de protestation, les autorités russes avaient gelé en juin 2017 leur budget accordé au Conseil de l'Europe ${ }^{14}$, d'un montant de 33000 euros.

La Russie fait d'ailleurs preuve de défiance face aux institutions européennes et particulièrement la Cour strasbourgeoise. En effet, le mardi 1er décembre 2015, la chambre basse du parlement russe, la Douma, a voté un texte de loi qui place la Cour constitutionnelle russe au-dessus de la Cour ESDH dans la hiérarchie des normes. Le texte précise que les arrêts de la Cour des droits de l'Homme ne s'appliqueront pas dès lors qu'ils seront déclarés inconstitutionnels par la Cour constitutionnelle russe. Il semblerait que la loi ait été adoptée suite à l'impopulaire jurisprudence Yukos ${ }^{15}$ de 2014. Cette affaire concerne les procédures fiscales de redressement et de recouvrement dirigées contre la société pétrolière russe OAO Neftyanaya Kompaniya YUKOS, et s'étant soldées par sa liquidation. La Cour a, à cette occasion, ordonné à la Russie de payer près de 1,9 milliard d'euros de dédommagement aux anciens actionnaires de l'ex-groupe pétrolier, pour des irrégularités dans la procédure fiscale intentée contre la société en 2000.

$\mathrm{Au}$ regard du caractère politique de l'arrêt de Grande Chambre "Navalnyy c. Russie", il semble y avoir de faibles chances que ce dernier soit executé par la Fédération de Russie. Toutefois, soulignons le Conseil de l'Europe devra chercher une issue à cette relation conflictuelle, sans quoi, un éloignement définitif de Moscou semble inévitable.

Cour.EDH, Gr.Ch., 15 novembre 2018, Navalny c. Russie, req. n²9580/12

Les Lettres « Actualités Droits-Libertés » (ADL) du CREDOF (pour s'y abonner) sont accessibles sur le site de la Revue des Droits de l'Homme (RevDH) 


\section{NOTES}

1. CEDH, arrêt de Chambre, Lashmankin et autres c. Russie, 7 février 2017, req. $n$ -57818/09

2. Rapport 2017/2018 Amnesty International, la situation des droits humains dans 159 pays en 2017

3. CEDH, arrêt de Grande Chambre, Navalnyy c. Russie, 15 novembre 2018, req. $n$ - $29580 / 12$

4. CEDH, Navalnyy c. Russie, 2 févr. 2017, req. $n^{\circ} 29580 / 12$

5. recueil des travaux préparatoires de la Convention européenne des droits de l'homme, Martinus Nijhoff, vol. IV, 1977, pp. 130, 179-181 et 955

6. $\$ \$ 303$ et 306 Merabishivili c. Georgie, 28 nov. 2017, req. $n^{\circ} 72508 / 13$

7. CEDH, gr. ch., 28 nov. 2017, Merabishvili c. Géorgie, req. $n^{\circ} 72508 / 13$

8. $\S \S 302$ et 303 Merabishivili c. Géorgie, 28 nov. 2017 , req. $n^{\circ} 72508 / 13$

9. §§ 167-168 Navalnyy c. Russie, 15 nov. 2018 , req. $n^{\circ} 29580 / 12$

10. Opinion partiellement dissidente aux juges Lòpez Guerra, Keller et Pastor Vilanova

11. CEDH, Lashmankin et autres c. Russie, 7 février 2017, req. $n^{\circ}$ 57818/09

12. Opinion en partie concordante, en partie dissidente, des juges Pejchal, Dedov, Ravarani, Eicke et Pacolay

13. Le point International, 'Entre la Russie et l'Europe, le spectre d'une crise durable' (Le Point International, 20 janvier 2018) <https://www.lepoint.fr/monde/entre-la-russie-et-leconseil-de-l-europe-le-spectre-d-une-crise-durable-20-01-2018-2188215_24.php>

14. Vladimir Vachtchenko, 'La Russie pourrait quitter le Conseil de l'Europe' (Le Courrier de Russie, 8 octobre 2018) <https://www.lecourrierderussie.com/international/2018/10/ quitter-conseil-europe/>

15. CEDH, Oao Neftyanaya Kompaniya Yukos c. Russie, 15 décembre 2014, n 14902/04

\section{RÉSUMÉS}

La présente lettre porte sur l'arrêt de Grande Chambre de la Cour européenne des droits de l'homme du 15 novembre 2018 condamnant la Fédération de Russie. Le requérant est le principal opposant politique de Vladimir Poutine, Alexey Navalnyy. Celui-ci avait été arrêté à sept reprises pour des rassemblements non autorisés et arguait de ce que ces détentions et arrestations administratives ont violé plusieurs de ses droits fondamentaux tels que son droit à la liberté de réunion pacifique, et étaient fondées sur un mobile politique. En l'espèce, la Cour conclut à une violation de l'article 18 en combinaison avec les articles 5 et 11 . La violation de l'article 18 avait été écartée par l'arrêt de chambre précédent. L'arrêt met ainsi en lumière l'existence d'une lutte arbitraire des autorités contre l'opposition politique en Russie. Cependant, l'opinion dissidente de certains juges laisse à penser qu'il aurait été loisible à la Cour de se placer sur le terrain de 
l'article 17 en ce qui concerne l'argument tiré de l'abus de droit. Au regard du caractère sensible de l'arrêt et des relations conflictuelles existant entre cet État et le Conseil de l'Europe, les chances d'exécution de l'arrêt apparaissent faibles, voire inexistantes.

\section{AUTEURS}

MARIA LESIRE

Étudiante du M2 Droits de l'Homme, Université Paris Nanterre

\section{ESTHER SEBILLOTTE}

Étudiante du M2 Droits de l'Homme, Université Paris Nanterre

\section{LISE SOUQUE}

Étudiante du M2 Droits de l'Homme, Université Paris Nanterre 